\title{
Balanced duo of anti-inflammatory SFRP5 and proinflammatory WNT5A in children
}

\author{
Anna Prats-Puig 1,2, Pilar Soriano-Rodríguez ${ }^{3}$, Gemma Carreras-Badosa ${ }^{1,2}$, Elena Riera-Pérez ${ }^{4}$, Monserrat Ros-Miquel${ }^{5}$, \\ Antoni Gomila-Borja ${ }^{5}$, Francis de Zegher ${ }^{6}$, Lourdes Ibáñez ${ }^{7,8}$, Judit Bassols ${ }^{1,2}$ and Abel López-Bermejo 1,2,9
}

BACKGROUND: Secreted frizzled-related protein 5 (SFRP5) is an adipokine protecting against obesity-related insulin resistance and diabetes. SFRP5 binds to wingless type mouse mammary tumor virus (MMTV) integration site family member 5A (WNT5A) to improve insulin sensitivity. We performed the first study of SFRP5 and WNT5A simultaneously in children.

METHODS: Prepubertal children $(n=342)$ were assessed for circulating SFRP5 (all subjects) and circulating WNT5A (210 subjects), and associations were sought with metabolic markers. In conditioned media of adipose tissue explants from 12 additional children, SFRP5 and WNT5A were studied further.

RESULTS: The concentrations of SFRP5 and WNT5A correlated positively in serum and in conditioned media (all $P<0.001$ ). Lower level of circulating SFRP5 (lowest quartile) was associated with higher BMI $(15 \%$ increase, $P<0.0001)$ and lower level of high-molecular-weight adiponectin (26\% decrease, $P=0.002$ ). Circulating WNT5A related closely with insulin resistance assessed by the homeostasis model assessment for insulin resistance and hepatic markers (alanine transaminase and gamma glutamyl transpeptidase), particularly in children with lower circulating SFRP5 levels (all $P<0.004$ ).

CONCLUSION: SFRP5 and WNT5A comprise a balanced duo that may regulate metabolic homeostasis in prepubertal children.

O besity is a common disorder that predisposes individuals to a number of metabolic abnormalities and is often associated with a low-grade inflammatory state in adipose tissue $(1,2)$. Adipose tissue constitutes the body's largest endocrine organ, secreting a variety of cytokines, referred to as adipokines (3).

Secreted frizzled-related protein 5 (SFRP5) is a recently described adipokine that has been associated with obesityrelated insulin resistance and type 2 diabetes (4-8). Systemic administration of SFRP5 to animals lacking SFRP5 gene improved glucose tolerance and insulin sensitivity and diminished inflammation (5). SFRP5 binds and sequesters wingless type mouse mammary tumor virus (MMTV) integration site family member $5 \mathrm{~A}$ (WNT5A) in the adipose tissue extracellular space. The Wnt proteins are a family of secreted glycoproteins that signal through a cell surface receptor known as frizzled. WNT5A could act as an important proinflammatory factor by upregulating the serine-threonine kinase c-Jun $\mathrm{N}$-terminal kinase $1(9,10)$. It is believed that SFRP5 binding to WNT5A attenuates Wnt signaling $(11,12)$, thereby restraining the chronic inflammatory state and improving insulin sensitivity (5). Recent studies suggested that WNT5A could also play a role in the activation of hepatic stellate cells, which has been related to the development of liver disease associated with insulin resistance $(13,14)$.

The SFRP5-WNT5A regulatory axis in fat may thus represent a potential target for the control of obesity-linked metabolic abnormalities (5). In a first study in children, we hypothesized that low SFRP5 levels would be related to a lessfavorable metabolic phenotype and would potentiate the association of WNT5A with insulin resistance.

\section{RESULTS}

To ascertain whether SFRP5 was associated with metabolic risk markers in prepubertal children, the studied subjects were stratified according to SFRP5 quartiles (Table 1). In general linear models, after adjusting for age, gender, and BMI, there were statistically significant differences for a number of metabolic characteristics among the quartile groups. Subjects in the lowest quartile of serum SFRP5 exhibited higher systolic blood pressure-standard deviation score (SDS) $(P=0.001)$, diastolic blood pressure (DBP)-SDS $(P=0.007)$, carotid intima-media thickness $(P<0.05)$, triglyceride-to-highdensity lipoprotein ratio $(P<0.01)$, homeostasis model assessment for insulin resistance (HOMA-IR) $(P=0.025)$, leptin $(P<0.0001)$, and high-sensitivity $\mathrm{C}$-reactive protein $(P=$ $0.002)$ and lower levels of high-density-lipoprotein cholesterol $(P<0.05)$ and high-molecular-weight adiponectin $(P=0.002)$,

\footnotetext{
'Pediatrics Research Group, Girona Institute for Biomedical Research, Girona, Spain; ${ }^{2}$ Department of Pediatrics, Dr Josep Trueta Hospital, Girona, Spain; ${ }^{3}$ Clinical Laboratory Salut Empordà Foundation, Figueres, Spain; ${ }^{4}$ Department of Pediatrics, Salut Empordà Foundation, Figueres, Spain; ${ }^{5}$ Department of Pediatric Surgery, Dr Josep Trueta Hospital, Girona, Spain; ${ }^{6}$ Department of Development \& Regeneration, University of Leuven, Leuven, Belgium; ${ }^{7}$ Department of Pediatric Endocrinology, Sant Joan de Déu Children's Hospital, Esplugues, Barcelona, Spain; ${ }^{8}$ CIBERDEM (Spanish Biomedical Research Centre in Diabetes and Associated Metabolic Disorders), Instituto de Salud Carlos III, Madrid, Spain; ${ }^{9}$ TransLab Research Group, Department of Medical Sciences, Faculty of Medicine, University of Girona, Girona, Spain. Correspondence: Abel López-Bermejo (alopezbermejo@idibgi.org)
} 
Table 1. Anthropometric and endocrine-metabolic variables in the studied subjects according to quartiles of SFRP5

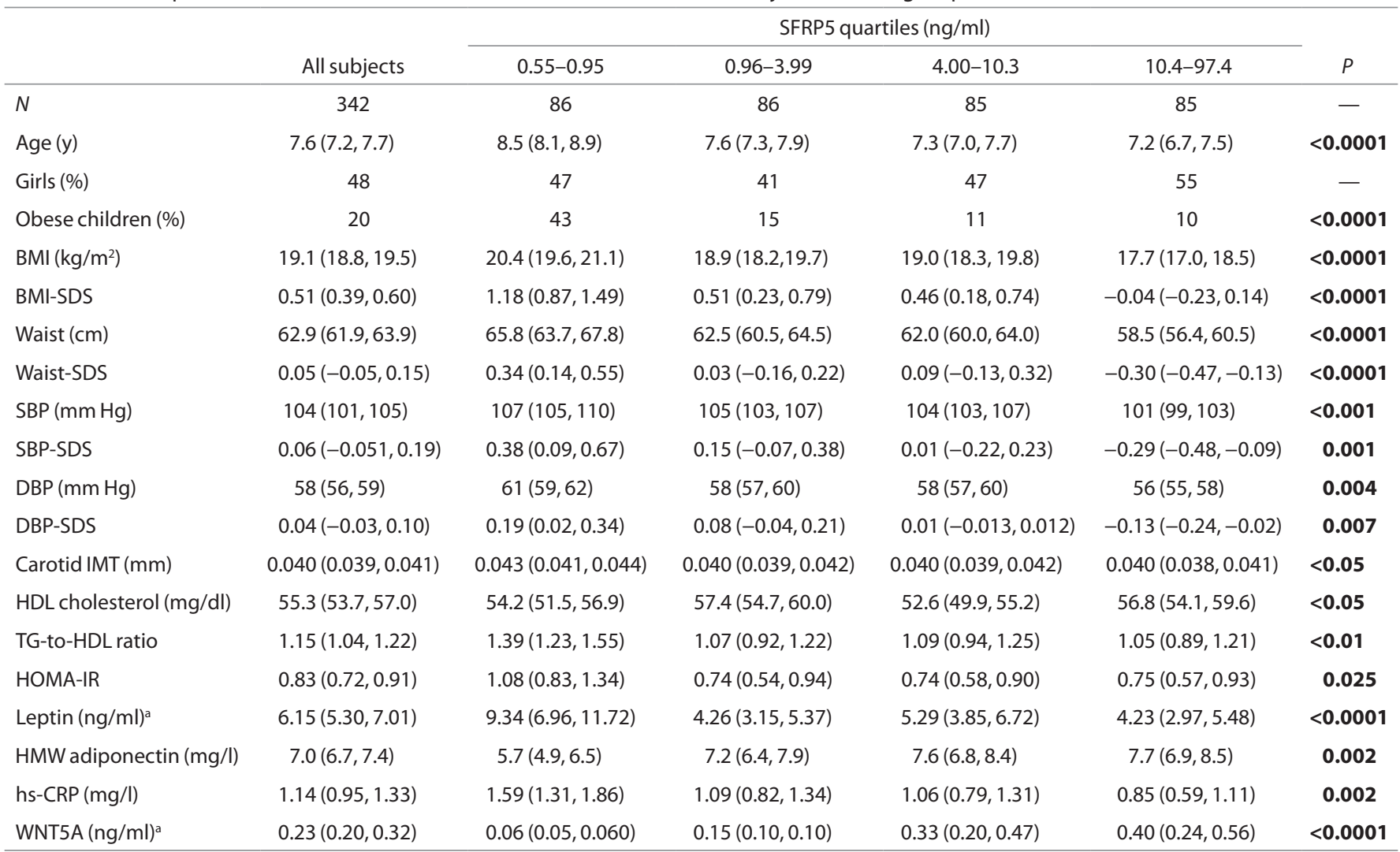

Data are shown as mean and interquartile range, adjusted for age, gender, and BMI in general linear models. Boldfaced data show significant results.

DBP, diastolic blood pressure; HDL, high-density lipoprotein; HMW, high-molecular weight; HOMA-IR, homeostasis model assessment for insulin resistance; hs-CRP, high-sensitivity

C-reactive protein; IMT, intima-media thickness; MMTV, mouse mammary tumor virus; SBP, systolic blood pressure; SDS, standard deviation score; SFRP5, secreted frizzled-related protein 5; TG, triglyceride; WNT5A, wingless type MMTV integration site family member 5 A.

${ }^{\mathrm{a}}$ Measured in a set of 210 prepubertal children.

compared with the levels in subjects in the highest quartile of serum SFRP5 (Table 1; Supplementary Figure S1 online). In multiple regression analyses, BMI-SDS and high-molecularweight adiponectin were independent predictors of SFRP5, together explaining $19.2 \%$ of its variance (Table 2). The inclusion or exclusion of obese subjects did not change the results of our study, and no gender interactions were found in any of the above-mentioned associations (results not shown).

Decreased SFRP5 levels were also associated with a significant increase in the prevalence of obesity after adjusting for age and gender (prevalence of obesity ranged from $10.5 \%$ in the highest quartile to $43.6 \%$ in the lowest quartile of SFRP5; $P<$ 0.0001 ). Consequently, children with lower levels of SFRP5 had an elevated odds ratio for obesity, after controlling for potential confounders $(P<0.0001$; Supplementary Figure $\mathbf{S} 2$ online).

SFRP5 and WNT5A protein levels were highly positively correlated $(P<0.0001)$ in serum from the studied subjects $(n=210)$ and in conditioned media from samples of visceral and subcutaneous adipose tissue $(n=12$; Supplementary Figure $\mathbf{S 3}$ online). The association between WNT5A and metabolic risk markers was therefore examined in children with lower and higher serum SFRP5 concentrations.

Serum WNT5A was related to higher insulin resistance (HOMA-IR: $r=0.236, P<0.001$ ) and hepatic markers (alanine

Table 2. Multivariate regression analysis with serum SFRP5 as dependent variable

$\beta$

All children $(n=342)$

SFRP5

BMI-SDS

HMW adiponectin (mg/l)

$P$

$P$

$R^{2}$

(19)

0.192

$R$-square shows the combined effect of the independent variables. Nonpredictive variables are age, gender, SBP, TG-to-HDL ratio, and HOMA-IR. Boldfaced data show significant results.

HDL, high-density lipoprotein; HMW, high-molecular weight; HOMA-IR, homeostasis model assessment for insulin resistance; SBP, systolic blood pressure; SDS, standard deviation score; SFRP5, secreted frizzled-related protein 5; TG, triglyceride.

transaminase (ALT): $r=0.127, P<0.05$ ) in prepubertal children. In children with lower SFRP5, WNT5A was strongly associated with (i) insulin resistance (HOMA-IR: $r=0.339, P<$ 0.001 ) and (ii) hepatic markers (ALT: $r=0.344, P<0.0001$; and gamma glutamyl transpeptidase: $r=0.264, P<0.001$ ), which was not apparent in children with higher SFRP5 concentrations (Table 3). No gender interactions were found in these associations (data not shown).

In multiple regression analyses, BMI-SDS and WNT5A were independently related to HOMA-IR and ALT (Table 4). The 
Table 3. Partial correlations of WNT5A with selected parameters

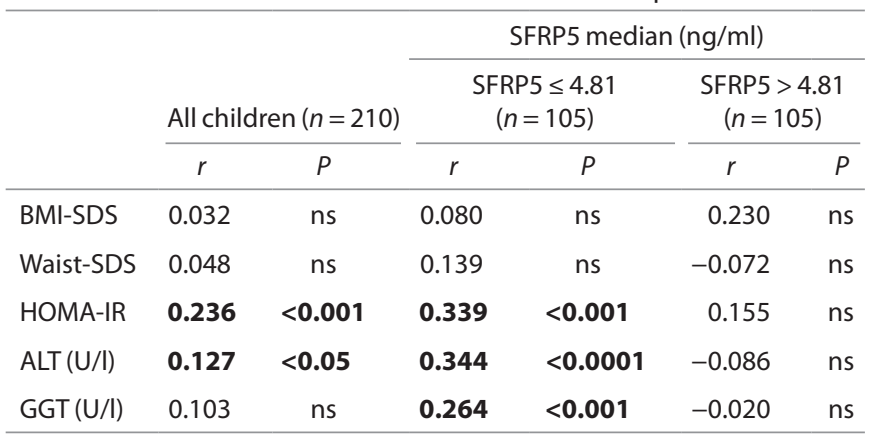

All results are adjusted for age. Boldfaced data show significant results.

ALT, alanine transaminase; GGT, gamma glutamyl transpeptidase; HOMA-IR,

homeostasis model assessment for insulin resistance; MMTV, mouse mammary tumor

virus; SDS, standard deviation score; SFRP5, secreted frizzled-related protein 5; WNT5A,

wingless type MMTV integration site family member $5 \mathrm{~A}$.

association with WNT5A was stronger in children with lower SFRP5, in whom BMI-SDS and WNT5A together explained $60.5 \%$ of HOMA-IR and $20.4 \%$ of ALT variance (Table 4 ).

\section{DISCUSSION}

Ours is the first report associating decreased concentrations of SFRP5 with obesity markers and lower level of high-molecular-weight adiponectin in prepubertal children. We also report that lower serum SFRP5 potentiated the association between WNT5A and insulin resistance.

SFRP5 is a recently described adipokine that has been associated with obesity-related insulin resistance and type 2 diabetes $(4-6,8)$. A recent study showed that circulating SFRP5 was decreased in patients with impaired glucose tolerance or type 2 diabetes and was associated with various obesity-related metabolic parameters $(4,7)$. SFRP5 sequesters WNT5A, thereby attenuating the activation of c-Jun N-terminal kinase $1(9,10)$. Our results suggest that SFRP5 may be an anti-inflammatory adipokine that could be negatively regulated during obesity development, leading to a less-favorable metabolic phenotype. For some of the metabolic parameters studied, the results may be indicative of a threshold effect, with a poorer metabolic profile restricted to subjects within the lower SFRP5 quartile.

We also describe that WNT5A was positively related to SFRP5 in both serum and conditioned media of adipose tissue explants. In children with lower SFRP5 serum levels, WNT5A was more strongly related to insulin resistance and hepatic markers, which fits with the known proinflammatory action of WNT5A on preadipocyte cells, preventing their differentiation and leading to insulin resistance (15). These results suggest that a failure to upregulate SFRP5 in obesity may lead to unrestrained proinflammatory actions of WNT5A, resulting in metabolic dysfunction $(5,6)$. It is well established that the inability to store the excess of energy in adipose tissue contributes to ectopic lipid accumulation in the liver (16). A recent study also reported that WNT5A is involved in the activation of hepatic stellate cells, which in turn is implicated in nonalcoholic fatty liver disease (13).

In our study, SFRP5 levels decreased with age in prepubertal children. It is well established that levels of adipokines such as
Table 4. Multivariate regression analyses using HOMA-IR and ALT as dependent variables

\begin{tabular}{lccc}
\hline & $\beta$ & $P$ & $R^{2}$ \\
\hline All children $(n=210)$ & & & \\
HOMA-IR & & & \\
BMI-SDS & 0.483 & $\mathbf{0 . 0 0 0 1}$ & 0.413 \\
WNT5A $(\mathrm{ng} / \mathrm{ml})$ & 0.131 & $\mathbf{0 . 0 3 8}$ & \\
ALT (U/l) & & & 0.114 \\
BMI-SDS & 0.291 & $\mathbf{0 . 0 0 1}$ & \\
WNT5A $(\mathrm{ng} / \mathrm{ml})$ & 0.214 & $\mathbf{0 . 0 0 5}$ & \\
SFRP5 $\leq 4.81 \mathrm{ng} / \mathrm{ml}(n=105)$ & & & 0.605 \\
HOMA-IR & & & \\
BMI-SDS & 0.715 & $<\mathbf{0 . 0 0 0 1}$ & \\
WNT5A $(\mathrm{ng} / \mathrm{ml})$ & 0.171 & $\mathbf{0 . 0 3 2}$ & \\
ALT (U/l) & & & \\
BMI-SDS & 0.361 & $\mathbf{0 . 0 1 9}$ & \\
WNT5A $(\mathrm{ng} / \mathrm{ml})$ & 0.330 & $\mathbf{0 . 0 0 3}$ &
\end{tabular}

$R$-square shows the combined effect of the independent variables. Nonpredictive variables are age, gender, TG-to-HDL ratio, and SFRP5; and for ALT, HOMA-IR also is included. Boldfaced data show significant results.

ALT, alanine transaminase; high-density lipoprotein; HOMA-IR, homeostasis model assessment for insulin resistance; MMTV, mouse mammary tumor virus; SDS, standard deviation score; SFRP5, secreted frizzled-related protein 5;WNT5A, wingless type MMTV integration site family member $5 \mathrm{~A}$.

adiponectin, a bona fide anti-inflammatory adipokine, decline in parallel with age and pubertal development in association with changes in growth factors (17) and androgen levels (18). Similarly, increased leptin level during puberty is a reliable indicator of insulin resistance associated with increasing age (19). Thus, we cannot exclude the possibility that reduced SFRP5 levels may contribute to the state of insulin resistance associated with increasing age and pubertal development (20). However, mores studies are needed in pubertal children to ascertain the influence of age and puberty on SFRP5 levels.

To the best of our knowledge, this is the first article to study SFRP5 and WNT5A simultaneously and the interaction thereof with metabolic risk markers, and this is also the first description in children in whom the metabolic abnormalities associated with obesity are known to originate. We acknowledge that our findings need to be corroborated in other populations and that longitudinal studies are warranted to disclose whether children with low levels of SFRP5 and/or unrestrained levels of WNT5A may be more likely to develop obesity-related metabolic dysfunction.

In summary, this exploratory study illustrates that SFRP5 and WNT5A comprise a balanced duo that may regulate metabolic homeostasis in prepubertal children. SFRP5 is a putative anti-inflammatory adipokine that could, at least in part, restrain the proinflammatory effects of WNT5A in children.

\section{METHODS}

\section{Population and Ethics}

Subjects were 342 school-aged Caucasian children (179 boys and 163 girls; age: $7.2 \pm 0.1 \mathrm{y}$ ) included in an ongoing study of cardiovascular risk factors in prepubertal children. Subjects were consecutively 
recruited among those seen in a primary care setting in Alt Emporda and Girona, both regions in Northern Spain. Clinical characteristics for part of this cohort have been recently reported (21).

Inclusion criteria were (i) age between 5 and $10 \mathrm{y}$ and (ii) no pubertal development, as judged by a specifically trained nurse using Tanner criteria (breast stage I; testicular volume $<4 \mathrm{ml}$ ). Exclusion criteria were (i) major congenital abnormalities; (ii) abnormal blood counts and abnormal liver, kidney, or thyroid functions; (iii) evidence of chronic illness or chronic use of medication; (iv) acute illness or use of medication in the month preceding potential enrollment; and (v) malnutrition, defined as BMI-SDS $\leq-2$.

The research was approved by the Institutional Review Board of Dr Josep Trueta Hospital. Signed consent was obtained from the parents.

\section{Clinical Assessments}

Clinical examination was performed in the morning, in the fasting state, followed by venous blood sampling. Weight was measured wearing light clothes with a calibrated scale, and height was measured with a Harpenden stadiometer. BMI was calculated as weight divided by the square of height in meters. Waist circumference was measured in the supine position at the umbilical level. Age- and sex-adjusted SDSs for BMI and waist circumference were calculated using regional normative data. Childhood obesity was defined as BMI-SDS $\geq 2$.

Blood pressure was measured in the supine position on the right arm after a 10-min rest; an electronic sphygmomanometer with cuff size appropriate for arm circumference was used. Age- and sexadjusted SDSs for waist circumference and blood pressure were calculated using our population data.

\section{High-Resolution Ultrasound Measurements}

Carotid intima-media thickness was measured by high-resolution ultrasonography. Diastolic images were obtained using a linear $12-\mathrm{MHz}$ transducer on the right side at the level of the distal common carotid artery, $1 \mathrm{~cm}$ away from its bifurcation point. Averages of five measurements of the carotid intima-media thickness on the far wall of the artery were used in the study. All measurements were taken on a separate visit in all children and were performed by the same observer who was unaware of the clinical and laboratory characteristics of the subjects. Intrasubject coefficient of variation $(\mathrm{CV})$ for ultrasound measurements was $<6 \%$. None of the children in our study showed ultrasonographic signs of atherosclerotic plaques.

\section{Laboratory Variables}

All serum samples were obtained between 8:00 and 9:00 AM under fasting conditions. Serum glucose was analyzed by the glucose hexoquinase method. Serum immunoreactive insulin was measured by immunochemiluminescence. Lower detection limit was $0.4 \mathrm{mIU} / \mathrm{l}$ and intra- and interassay CVs were $<10 \%$. Insulin resistance was calculated using the HOMA-IR (HOMA-IR = (fasting insulin, in mU/l) $\times$ (fasting glucose, in $\mathrm{mmol} / \mathrm{l}$ )/22.5). High-density-lipoprotein cholesterol was quantified by homogenous method of selective detergent with accelerator. Total serum triglycerides were measured by monitoring the reaction of glycerol phosphate oxidase. Serum aspartate transaminase, ALT, and gamma glutamyl transpeptidase were measured by colorimetry using automated tests. Intra- and interassay CVs were $<4 \%$ for these tests. Serum levels of high-sensitivity C-reactive protein were measured using the ultrasensitive latex immunoassay CRP Vario (Sentinel Diagnostics, Milan, Italy). Lower detection limit was $0.2 \mathrm{mg} / \mathrm{l}$, and the intra- and interassay CVs were $<3 \%$. All values $<0.2 \mathrm{mg} / \mathrm{l}$ were coded as 0.2 . Children with highsensitivity C-reactive protein values $>10.0 \mathrm{mg} / \mathrm{l}$ were excluded from the study.

High-molecular-weight adiponectin was measured by sandwich enzyme-linked immunosorbent assay (Linco, St Charles, MO), the detection limit was $0.5 \mathrm{ng} / \mathrm{ml}$, and the $\mathrm{CV}$ was $<4 \%$. Leptin was measured by sandwich enzyme-linked immunosorbent assay (Linco), the detection limit was $0.2 \mathrm{ng} / \mathrm{ml}$, and the CV was $<3 \%$. SFRP5 and WNT5A were measured by sandwich enzyme immunoassays (Uscn Life Science, Wuhan, People's Republic of China), with sensitivities of 0.55 and $0.053 \mathrm{ng} / \mathrm{ml}$, respectively. CVs at our laboratory were $<6 \%$. The leptin and WNT5A assessments were limited to a representative randomly selected sample of the studied subjects whose clinical characteristics did not diverge from those of the whole group ( $n=210 ; 115$ boys and 95 girls).

\section{Adipose Tissue Explants}

Samples of visceral and subcutaneous adipose tissue biopsies were obtained after obtaining written informed consent from 12 additional children ( 10 boys and 2 girls, age: $6.1 \pm 1.3 \mathrm{y}$ ) undergoing abdominal surgery for urological malformations or repair of inguinal hernia. All subjects were otherwise asymptomatic (they were free from any acute or chronic illness) and had normal blood counts and normal liver and kidney functions. Adipose tissue biopsies were cut into small pieces and washed in PBS with 5\% bovine serum albumin for $30 \mathrm{~min}$ at room temperature. Explants were incubated $(\sim 300 \mathrm{mg}$ of tissue per well) in 12-well plates with $1 \mathrm{ml}$ Dulbecco's modified essential medium supplemented with $1 \%$ sodium pyruvate, $1 \%$ glutamine, $100 \mathrm{U} / \mathrm{ml}$ penicillin, and $0.1 \mathrm{mg} / \mathrm{ml}$ streptomycin for up to $48 \mathrm{~h}$ at 37 ${ }^{\circ} \mathrm{C}$ in $5 \% \mathrm{CO}_{2}$. Conditioned media were collected for studies of concentrations of total protein, SFRP5, and WNT5A at $48 \mathrm{~h}$.

Both SFRP5 and WNT5A were measured in conditioned media using the same commercial assays as described above. Results were adjusted for the sum of total protein concentration in conditioned media and in tissue lysate, as measured by the Lowry method (22).

\section{Assessment of Viability of Adipose Tissue Explants}

Viability of adipose tissue explants was assessed by measuring lactate dehydrogenase activity in conditioned media. To prepare for the lactate dehydrogenase assay, the conditioned medium of adipose tissue was transferred to a $1.5-\mathrm{ml}$ microcentrifuge tube and centrifuged at $12,000 \mathrm{~g}$ and $4^{\circ} \mathrm{C}$ for $15 \mathrm{~min}$ to remove cell debris.

Measurement of total lactate dehydrogenase activity in the samples was obtained by the International Federation of Clinical Chemistry and Laboratory Medicine-recommended forward reaction (23), which catalyzes the oxidation of $\mathrm{L}$-lactate to pyruvate with the mediation of oxidized nicotinamide adenine dinucleotide as a hydrogen acceptor (Architect, Abbott Diagnostics, Milan, Italy). Intra- and interassay CVs were $<4.7 \%$ for this test. Results were adjusted for the sum of total protein concentration in conditioned media and in tissue lysate, as measured by the Lowry method (22).

\section{Statistics}

Statistical analyses were performed using SPSS version 12.0. Results are expressed as mean and 95\% CI. Nonparametric variables were logarithmically transformed to improve symmetry. Differences among SFRP5 quartiles in children were evaluated using general linear models after adjusting for age, gender, and BMI. Partial correlation followed by multiple regression analyses were used to analyze the associations of SFRP5 and WNT5A with metabolic parameters. The enter method was used for computing the independent variables. Logistic regression analyses were used to identify independent associations between SFRP5 quartiles and obesity. Significance level was set at $P<0.05$. The sample size was calculated to have $80 \%$ power to detect significant correlations between circulating SFRP5 or WNT5A and metabolic risk markers, with a Pearson correlation coefficient of at least 0.30 in both total and subgroups analyses $(n=84$ would be the minimum sample size in subgroup analyses in order to detect such correlations in bilateral tests).

\section{SUPPLEMENTARY MATERIAL}

Supplementary material is linked to the online version of the paper at http://www.nature.com/pr

\section{ACKNOWLEDGMENTS}

We are grateful to all the children and parents who took part in the study.

\section{STATEMENT OF FINANCIAL SUPPORT}

This work was supported by grant no. 10/00553 from Carlos III National Institute of Health, Madrid, Spain (Fondo de Investigaciones Sanitarias (FIS) Fund for Health Research) to A.L.-B.). G.C.-B. is an investigator of the Predoctoral Fund for Health Research from Carlos III National Institute of Health, Madrid, Spain (FIS Fund for Health Research). F.d.Z. is a clinical investigator of the Fund for Scientific Research), Flanders, Belgium. L.I. is a clinical investigator of CIBERDEM, Madrid, Spain (Spanish Biomedical 
Research Centre in Diabetes and Associated Metabolic Disorders), and J.B. is an investigator of the Miguel Servet Fund from Carlos III National Institute of Health, Madrid, Spain (FIS Fund for Health Research). A.L.-B. is an investigator of the 13 Fund for Scientific Research, Madrid, Spain (Ministry of Science and Innovation).

Disclosure: The authors declared no conflicts of interest.

\section{REFERENCES}

1. Ode KL, Frohnert BI, Nathan BM. Identification and treatment of metabolic complications in pediatric obesity. Rev Endocr Metab Disord 2009;10:167-88.

2. Goldfine AB, Fonseca V, Shoelson SE. Therapeutic approaches to target inflammation in type 2 diabetes. Clin Chem 2011;57:162-7.

3. Spiegelman BM, Flier JS. Adipogenesis and obesity: rounding out the big picture. Cell 1996;87:377-89.

4. Hu Z, Deng H, Qu H. Plasma SFRP5 levels are decreased in Chinese subjects with obesity and type 2 diabetes and negatively correlated with parameters of insulin resistance. Diabetes Res Clin Pract 2013;99:391-5.

5. Ouchi N, Higuchi A, Ohashi K, et al. Sfrp5 is an anti-inflammatory adipokine that modulates metabolic dysfunction in obesity. Science 2010;329:454-7.

6. Schulte DM, Müller N, Neumann K, et al. Pro-inflammatory wnt5a and anti-inflammatory SFRP5 are differentially regulated by nutritional factors in obese human subjects. PLoS One 2012;7:e32437.

7. Hu W, Li L, Yang M, et al. Circulating Sfrp5 is a signature of obesity-related metabolic disorders and is regulated by glucose and liraglutide in humans. J Clin Endocrinol Metab 2013;98:290-8.

8. Carstensen M, Herder C, Kempf K, et al. Sfrp5 correlates with insulin resistance and oxidative stress. Eur J Clin Invest 2013;43:350-7.

9. Bilkovski R, Schulte DM, Oberhauser F, et al. Role of WNT-5a in the determination of human mesenchymal stem cells into preadipocytes. J Biol Chem 2010;285:6170-8.

10. Almind K, Kahn CR. Genetic determinants of energy expenditure and insulin resistance in diet-induced obesity in mice. Diabetes 2004;53:3274-85.

11. Bovolenta P, Esteve P, Ruiz JM, Cisneros E, Lopez-Rios J. Beyond Wnt inhibition: new functions of secreted Frizzled-related proteins in development and disease. J Cell Sci 2008;121(Pt 6):737-46.
12. Kawano Y, Kypta R. Secreted antagonists of the Wnt signalling pathway. J Cell Sci 2003;116(Pt 13):2627-34.

13. Atzori L, Poli G, Perra A. Hepatic stellate cell: a star cell in the liver. Int J Biochem Cell Biol 2009;41:1639-42.

14. Xiong WJ, Hu LJ, Jian YC, et al. Wnt5a participates in hepatic stellate cell activation observed by gene expression profile and functional assays. World J Gastroenterol 2012;18:1745-52.

15. Bilkovski R, Schulte DM, Oberhauser F, et al. Adipose tissue macrophages inhibit adipogenesis of mesenchymal precursor cells via wnt-5a in humans. Int J Obes (Lond) 2011;35:1450-4.

16. Wang CP, Chung FM, Shin SJ, Lee YJ. Congenital and environmental factors associated with adipocyte dysregulation as defects of insulin resistance. Rev Diabet Stud 2007;4:77-84.

17. Butte NF, Comuzzie AG, Cai G, Cole SA, Mehta NR, Bacino CA. Genetic and environmental factors influencing fasting serum adiponectin in Hispanic children. J Clin Endocrinol Metab 2005;90:4170-6.

18. Böttner A, Kratzsch J, Müller G, et al. Gender differences of adiponectin levels develop during the progression of puberty and are related to serum androgen levels. J Clin Endocrinol Metab 2004;89:4053-61.

19. Xu L, Li M, Yin J, et al. Change of Body Composition and Adipokines and Their Relationship with Insulin Resistance across Pubertal Development in Obese and Nonobese Chinese Children: The BCAMS Study. Int J Endocrinol 2012;2012:389108.

20. Bush NC, Darnell BE, Oster RA, Goran MI, Gower BA. Adiponectin is lower among African Americans and is independently related to insulin sensitivity in children and adolescents. Diabetes 2005;54:2772-8.

21. Prats-Puig A, Grau-Cabrera P, Riera-Pérez E, et al. Variations in the obesity genes FTO, TMEM18 and NRXN3 influence the vulnerability of children to weight gain induced by short sleep duration. Int J Obes (Lond) 2013;37:182-7.

22. Lowry OH, Rosebrough NJ, Farr AL, Randall RJ. Protein measurement with the Folin phenol reagent. J Biol Chem 1951;193:265-75.

23. Schumann G, Bonora R, Ceriotti F, et al. IFCC primary reference procedures for the measurement of catalytic activity concentrations of enzymes at 37 degrees C. Part 3. Reference procedure for the measurement of catalytic concentration of lactate dehydrogenase. Clin Chem Lab Med 2002;40:643-8. 